Reprod. Nutr. Dévelop., 1987, 27 (1 B), 265-266.

\title{
Relation entre la solubilité et la dégradation in sacco de l'azote
}

P. CHAPOUTOT, S. HAKIM, D. SAUVANT

avec la collaboration technique de Michelle DORLEANS, S. LEFRANÇOIS

Station de Nutrition et Alimentation (I.N.R.A.)

Institut National Agronomique Paris-Grignon, 16, rue Claude-Bernard, 75231 Paris Cedex 05.

Summary. Nitrogen solubility and $\mathrm{N}$ disappearance from nylon bags after $6,12,24$ and $48 \mathrm{~h}$ of rumen incubation were measured with 82 feedstuffs (concentrates and forages). The correlation coefficient between $\mathrm{N}$ solubility and $\mathrm{N}$ degradation was the highest after $6 \mathrm{~h}$ rumen incubation ( $R=0.76 ; E T R=13.7$ ) and decreased as incubation time increased. Effective nitrogen degradation $\left(0.06 \mathrm{~h}^{-1}\right.$ rate of passage), was more precisely predicted by solubility $(R=0.64 ; E T R=10.3)$ than degradation at different times of incubation. Therefore, the between-feeds hierarchies measured with these two methods were not the same.

La solubilité de la fraction azotée des aliments est une caractéristique prédictrice importante de la valeur azotée des aliments (Vérité et Demarquilly, 1978 ; Vérité et Sauvant, 1981). Au cours des dernières années, des études ont montré l'intérêt de la mesure de la dégradation in sacco de l'azote pour prévoir sa dégradation in vivo (Stern et Satter, 1984). Le travail présenté a pour but de préciser, sur un ensemble diversifié d'aliments, la liaison entre les mesures de solubilité et de dégradation in sacco de l'azote.

Matériel et méthodes. L'étude a porté sur 82 aliments : 16 aliments composés, 14 sous-produits de céréales, 11 foins de luzerne, 6 ensilages de maïs, 7 pulpes de betteraves normales et 12 enrichies (mélasse et/ou vinasse avec urée), 4 tourteaux, 2 céréales, 2 protéagineux, 3 sous-produits du raisin, 1 luzerne déshydratée normale et 3 enrichies en urée, 1 tégument de soja. Les échantillons ont été étudiés et analysés en l'état sauf les aliments composés, les foins de luzerne et les ensilages de maïs (séchage à l'étuve à $80^{\circ} \mathrm{C}$ ) et ont fait l'objet d'une mesure de la teneur en azote (Kjehldal) et de la solubilité de l'azote selon la méthode proposée par M. Durand (Vérité et Demarquilly, 1978). La mesure de la dégradation in sacco (Gueneau et Bertrand, 1984) a été effectuée sur des chèvres, porteuses de canules de rumen et recevant une ration comprenant 75 à $80 \%$ de foin de luzerne et 20 à $25 \%$ d'aliment concentré composé, de taux azoté variant de 15 à $18 \%$ de la M.S. Les sachets (dimensions internes $=9 \times 14 \mathrm{~cm}$ ) en tissu de nylon soudé (Réf. : F 100 ; Tripette et Renaud) contenaient $3 \mathrm{~g}$ d'aliment. Chaque aliment a fait l'objet de 3 à 6 répétitions. Pour chaque cinétique $(6,12,24$ et $48 \mathrm{~h}$ d'incubation) un même témoin (paille d'avoine) retiré après $24 \mathrm{~h}$ d'incubation permet de corriger par analyse de co-variance, les valeurs individuelles de dégradation de la M.S. Les résidus d'incubation sont regroupés par aliment et par temps pour les analyses d'azote.

Résultat et discussion. Les teneurs en azote des 82 aliments ont été comprises entre 1,2 et $7,8 \% \mathrm{MS}$ et la solubilité ( $\mathrm{N}$ soluble : $\mathrm{N}$ total) entre 3 et $77 \%$. La relation la plus étroite entre la solubilité et la dégradation in sacco de $\mathrm{N}$ est observée après $6 \mathrm{~h}$ d'incubation $(R=0,76$; ETR $=13,7$, fig. 1$)$; pour les durées plus longues la valeur du coefficient de corrélation diminue mais celle de l'écart-type résiduel varie peu $(R=0,63,0,36,0,24$ et $E T R=14,4,14,5,11,6$ respectivement à 12, 24 et 48 h). Crawford et al. (1978), Stern et Satter (1984), De Boever et al. (1984) ont également observé une diminution de la corrélation solubilitédégradation in sacco lorsque la durée d'incubation augmente. En outre, pour les 
deux premiers cités, les coefficients des régressions linéaires calculées sont très comparables aux nôtres, à temps égal d'incubation. Cette évolution de la relation solubilité-dégradation traduit, d'une part, la relation positive et étroite, entre les fractions azotées solubles et celles disparaissant rapidement des sachets et, d'autre part, la relation négative entre la solubilité et la proportion d'azote lentement dégradée $(R=-0,63$, fig. 2 ). Pour chaque aliment, la dégradabilité théorique de l'azote (D.T.) a été calculée à partir des valeurs de dégradation mesurées pour les 4 prélèvements : $6,12,24$ et $48 \mathrm{~h}$ (ti) en supposant une valeur initiale $\mathrm{D}_{0}=0$ pour $\mathrm{t}_{0}=0$ et un taux de renouvellement des particules dans le réticulo rumen $\mathrm{K}=0.06^{-1}$, d'après la formule suivante :

$$
\text { D. T. }=\sum_{i=1}^{i=4}\left(D_{i}-D_{i-1}\right) \frac{\left(e^{-k t_{i}}+e^{-k t_{i}-1}\right)}{2} \text {. }
$$

Les valeurs obtenues sont comprises entre 17,9 et $81,8 \%$. Elles sont prédites avec une meilleure précision que celles de la dégradation in sacco aux différents temps d'incubation ( $R=0,64, E T R=10,3$ ).
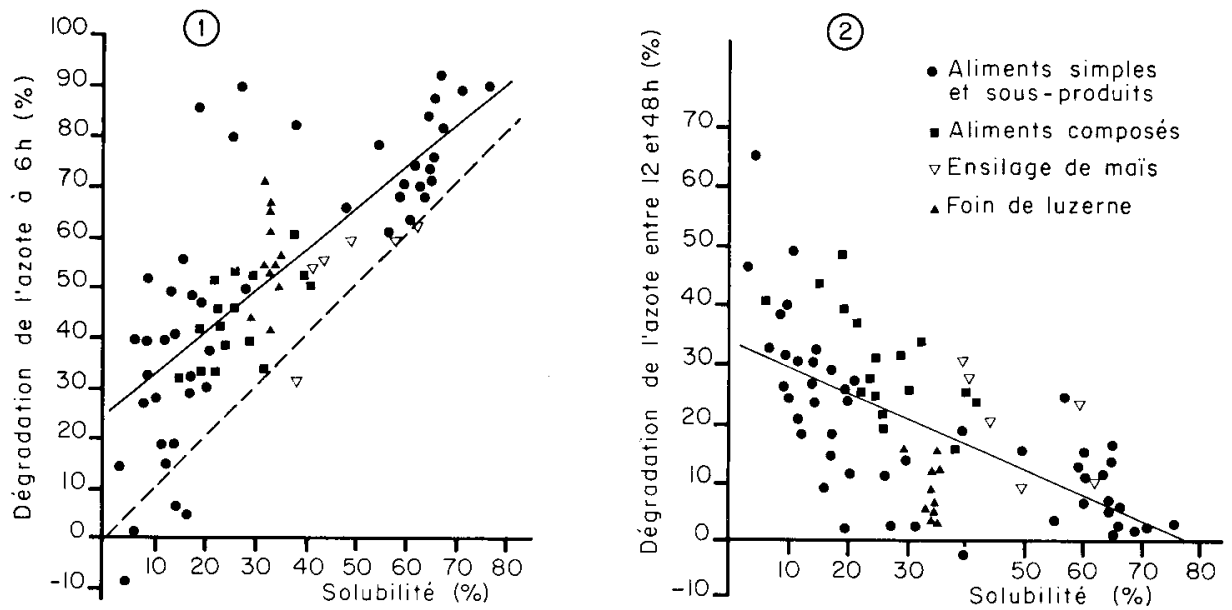

FIG. 1. - Relation entre la solubilité et la dégradation in sacco $(6$ h) de l'azote.

FIG. 2. - Relation entre la solubilité et la dégradation in sacco de l'azote entre 12-48 h.

Des sous-groupes de matières premières à caractéristiques communes se distinguent au sein des différentes relations calculées. Ainsi, l'orge, le blé et ses sous-produits présentent, par rapport à leur solubilité, des valeurs importantes de dégradation à court terme, tandis que des produits tels que les drêches de brasserie, les sous-produits de distillerie du maïs, les pulpes de raisin et de betteraves normales présentent de faibles valeurs de dégradation in sacco par rapport à la relation générale (figure 1). Ces résultats vont dans le sens des mesures in sacco de De Boever et al. (1984), de Crawford et al. (1978) et même de celles de Vérité et Sauvant (1981) obtenues par fermentescibilité in vitro (Vérité et Demarquilly, 1978).

Crawford R. H., Hoover W. H., Sniffen C. J., Crooker B. A., 1978. J. Anim. Sci., 46, $1768-1775$. De Boever J. L., Aerts J. V., Cottyn B. G., Vamacker J. M., Buysse F. X., 1984. Z. Tierphysiol, Tierernährg. u. Futtermittelkde, 52, 227-234.

Gueneau S., Bertrand D., 1984. Cahier des techniques de I'INRA, 4, 33-38.

Stern M. D., Satter L. D., 1984. J. anim. Sci., 58, 714-724.

Vérité R., Demarquilly C., 1978. In La vache laitière, pp. 143-157. Ed. INRA Publ., 78000 Versailles.

Vérité R., Sauvant D., 1981. In Prevision de la valeur nutritive des aliments des Ruminants, pp. 279 296, Ed. INRA Publ., 78000 Versailles. 